\title{
Treatment of behavioral symptoms and unsettled relationships in clinically complex residents in the long-term care facility
}

\author{
Urszula Horwath ${ }^{1,3} \cdot$ Katarzyna Szczerbińska ${ }^{2}$
}

Received: 31 July 2018 / Accepted: 1 November 2018 / Published online: 13 November 2018

(c) The Author(s) 2018

\begin{abstract}
Purpose Multifactorial etiology of behavioral symptoms (BS) and unsettled relationships (UR) in long-term care facility (LTCF) residents, who are characterized by comorbidity of somatic and mental diseases, makes the treatment process particularly difficult. The main goal of this study was to analyze a line of pharmacological and non-pharmacological treatment of clinically complex LTCF residents presenting BS and UR.

Methods This was a cross-sectional study of 301 LTCF residents with the use of interRAI-LTCF questionnaire. Descriptive statistics provided frequencies of pharmacological and non-pharmacological interventions in residents presenting BS and UR. Logistic regression models identified potential factors associated with administering antipsychotics, sedatives and antidepressants.

Results We found that residents with BS had a higher chance of being prescribed antipsychotics (OR 3.06; CI 1.59-5.86; $p<0.001)$. They were at greater risk of using sedatives only when BS were associated with older age or depression symptoms. However, BS associated with depression did not increase chance of using antidepressants. The UR had no impact on the use of psychotropic drugs. Moreover, staff's frustration in caring for residents with BS was associated with reduced risk of prescribing sedatives (OR 0.10 ; CI $0.02-0.68 ; p<0.02$ ). In contrast to pharmacotherapy, the non-pharmacological interventions were rarely applied.

Conclusions LTCF residents manifesting BS were more frequently treated with antipsychotics and sedatives compared to antidepressants, even when BS were associated with depression. The multifactorial etiology of BS and UR in a clinically complex LTCF resident requires recognition of the causes of BS and UR for an appropriate treatment to be applied.
\end{abstract}

Keywords Psychotropic drugs · Non-pharmacological interventions · Behavioral symptoms · Unsettled relationships . Elderly $\cdot$ Nursing home

\section{Abbreviations}

ADL Activity of daily living

ADLh Activities of daily living hierarchy scale

BS Behavioral symptoms

Electronic supplementary material The online version of this article (https://doi.org/10.1007/s41999-018-0127-2) contains supplementary material, which is available to authorized users.

Katarzyna Szczerbińska

katarzyna.szczerbinska@uj.edu.pl

Long-term Care Facility, Wielicka 267, Kraków, Poland

2 Unit for Research on Ageing Society, Department of Sociology of Medicine, Epidemiology and Preventive Medicine Chair, Medical Faculty, Jagiellonian University Medical College, Ul. Kopernika 7a, 31-034 Kraków, Poland

3 Medical Faculty, Jagiellonian University Medical College, Kraków, Poland
CPS Cognitive performance Scale

DRS Depression rating scale

LTC Long-term care

LTCF Long-term care facility

PT Physiotherapy

OT Occupational therapy

UR Unsettled relationships

\section{Introduction}

A long-term care facility (LTCF) is a specific setting where problems with social functioning are often presented by residents posing threat to themselves, other residents as well as their informal and formal caregivers. Prevalence of behavioral symptoms (BS, understood as a person's inappropriate verbal, vocal or motor activity [1-3]) and unsettled 
relationships (UR, defined as a reasonably consistent pattern of resident's hostility or criticism expressed verbally or with physical gestures toward other resident or staff member [4]) in LTCF varies widely from 7 to $91 \%$, depending on terminology $[1,5-8]$. The definitions of BS and UR used in this study are provided in the Annex. The occurrence of BS or UR in LTCF is a common reason for using physical or chemical restraints on residents and is associated with increased health care costs due to staff turnover, psychological workload and absenteeism $[8,9]$.

Based on literature review, the psychotropic drugs are often the first-line interventions used by health care professionals to treat BS and UR in older people $[8,10]$. The prevalence of prescribing antipsychotics amongst the older population is impressive. They are administered to the range of $17-65 \%$ of LTCF residents [11], despite the evidence indicating that these drugs have limited impact on reducing agitated and disruptive behaviors $[8,10,11]$. Moreover, the antipsychotic medicines are not recommended to older people for long-term use due to possible side effects $[8,11]$. Benzodiazepines are the second type of drugs most commonly prescribed to manage BS in older individuals. As the literature review indicates, benzodiazepines should also be used with caution among older residents, as their long-term administration has been linked with cognitive deterioration, poor functional autonomy, falls, and addictions [8, 10, 12]. On the other hand, antidepressant drugs are rarely chosen as treatment for BS among the older population [11].

Managing the BS and UR also involves non-pharmacological strategies. The review made by Cohen-Mansfield et al. [13] mentioned that social contact (real or simulated), psychological interventions (such as behavior therapy, reality orientation, validation therapy, life reviews), structured activities and multisensory therapy resulted in a statistically and clinically meaningful reduction of the manifestation of BS. Another review made by Snowden et al. [11] showed that music therapy, art therapy, ADL training, recreational therapy and a walking program are valuable in minimizing disruptive behaviors such as agitation and aggression. Other researchers pointed to the beneficial effect of physical exercise on reducing the frequency of problems in social functioning presented by LTCF residents [11,14-16]. When a non-pharmacological intervention is applied individually to each resident, the impact on BS is even greater $[11,13,17]$.

So far, there is extensive research literature on pharmacological and non-pharmacological interventions that addresses managing of BS and UR in patients with dementia. However, in many health care systems, the typical LTCF population consists of residents with a variety of physical and psychological health problems in the course of a chronic somatic and/or mental illness. Thus, in this paper, we aim to analyze the line of pharmacological and non-pharmacological treatment of clinically complex LTCF residents presenting BS (including wandering, physical and verbal abuse, socially and sexually inappropriate behavior, resistance to prescribed care or treatment), UR (such as conflict with or repeated criticism of other residents or staff) or a specific type of UR that is staff's persistent frustration during contact with a resident.

\section{Methods}

\section{Setting and Sample}

The study was performed within LTCF in a large city in Poland. It is an institution providing round-the-clock medical care (provided by a full-time employed physicians) and nursing care to 510 residents with physical and mental disability associated not only with dementia but also with a variety of somatic and psychiatric health problems. This particular LTCF provides care through three different departments: psychogeriatric ward (for residents with a chronic psychiatric disorder and Alzheimer's disease), palliative care ward (for patients in the terminal stage of cancer disease) and long-term care (LTC) ward (for disabled people with chronic somatic comorbidities). The aim of our study was to find the possible connections between certain treatments and problems with social functioning which are not directly related to severe psychiatric illness or end-of-life stage. Therefore, we decided to conduct the study only in the LTC ward for chronically ill residents to examine a group with mixed health problems and treatments, and thus to be able to notice differences between them. From a total of 354 residents admitted to the LTC ward, we excluded fifty-two residents who were unable to present any BP or UR due to coma or lack of discernible consciousness. One exclusion was on account of lack of data on analyzed factors. The final sample involved 301 residents aged 31-94 years, with 60 individuals below the age of $65(19.9 \%)$ and 82 individuals above the age of 85 (27.2\%). In Poland, the criterion for admission to LTCF is not age, but the Barthel Index below 40 points. That explains the relatively high frequency of people under 65 in the studied population. Since problems with social functioning are not limited only to older residents, we decided to study all clinically complex residents of the LTC ward to analyze therapeutic approaches in controlling their functioning.

\section{Study design}

This was a cross-sectional study. The LTC residents were assessed in 2013 during an 8 -month period by nurses who passed standardized training which included step-bystep assessments as specified in the user's manual of the interRAI-LTCF questionnaire (interRAI Long-Term Care 
Facilities Assessment System-www.interrai.org). The nurses completed the questionnaire based on a three-day observation of the residents as well as information obtained from medical records, family members and other staff.

The interRAI-LTCF is a comprehensive geriatric assessment tool which was designed to assist clinicians in supervising the care given to the LTCF residents and to provide a comprehensive view of the residents' health needs and strengths in a long-term care institution. The questionnaire includes over 350 variables reflecting socio-demographic characteristics, health status in terms of disease diagnoses, cognition, mood, behavior, functional status, medication, social support, and service use. It has been validated and proved to be reliable in several countries [18]. The questionnaire has been translated into Polish and passed cross-cultural adaptation preserving the rigorous format of translation methodology. The interRAI-LTCF tool comprises of several validated scales. A seven-point Cognitive Performance Scale (CPS) was used in the assessment of cognitive functioning as normal or nearly intact (score of $0-1$ ), moderately impaired (2-3) or severely impaired (4-6) [19]. Depression symptoms were evaluated using the seven-item Depression Rating Scale (DRS), where a score of 3 or higher suggested probable depression [20]. A seven-point Activities of Daily Living Hierarchy scale (ADLh) categorized the level of resident's self-reliance as independent (ADLh $=0-1)$, moderately dependent $(\mathrm{ADLh}=2-3)$, and severely dependent $(\mathrm{ADLh}=4-6)$ [21]. A score of Pain Scale ranged from 0 to 4 , with values equaling 2 and higher indicating daily presence of pain [22].

In our analysis, the problems with social functioning presented by LTCF residents were assessed in terms of presence of behavioral symptoms-BS (identified as any of the following: wandering, verbal or physical abuse, socially inappropriate behavior, inappropriate sexual behavior, and resistance to prescribed care or treatment), unsettled relationships-UR (such as conflict with or repeated criticism of other residents, conflict with or repeated criticism of staff) or specific type of UR that is staff reporting persistent frustration in dealing with resident. The current psychotic symptoms were defined as presence of delusions, hallucinations or abnormal thought process. Due to low prevalence of some psychiatric diseases, such as anxiety disorder, bipolar disorder and schizophrenia, we aggregated them in one variable of "psychiatric disorders other than depression and dementia".

Pharmacological interventions were recorded by the nurses based on drug dispensary cards which included the name and daily dose of drugs administered on the day of data collection. For statistical analysis, The Anatomical Therapeutic Chemical (ATC) was applied to classify psychotropic drugs into following groups: antipsychotics (N05AA, N05AB, N05AD, N05AF, N05AH, N05AL, N05AX), antidepressants (N06AA, N06AB, N06AX), and sedatives (including anxiolytics and hypnotics from the following ATC groups: N05BA, N05BB, N05CD, N05CF) [23]. The non-pharmacological interventions were categorized into four groups: physiotherapy (PT, physical therapy provided by a qualified physical therapist), occupational therapy (OT, individual and group therapy provided by a qualified occupational therapist aiming to engage residents in meaningful activities such as art and music therapy), psychological care (therapy tailored to the resident's needs given by a qualified psychologist) and ADL training (nursing intervention that promotes a person's ability to attain maximum functional potential in daily activities). The descriptions of each of the non-pharmacological intervention used in this particular LTCF and included in the analyses of this study are provided in the Annex.

In this particular LTCF, the residents were qualified for non-pharmacological interventions based on medical consultation (in the field of physiotherapy and ADL training) and psychologist examination (in the field of occupational therapy and psychological care). The non-pharmacological interventions as well as the diagnoses (coded with ICD-10) were obtained by the nurses from the medical records.

\section{Statistical analysis}

We applied the descriptive statistics including frequencies and percentages for qualitative variables, and for quantitative variables means and SDs for normally distributed ones, and medians and quartiles otherwise. We used Chi square test to examine frequency of pharmacological and nonpharmacological interventions in LTCF residents presenting BS, UR and other symptoms which might be associated with the occurrence of BS and UR. These were: age, gender, cognitive impairment and ADL dependency, psychotic symptoms (e.g., abnormal thought process, delusions, hallucinations), symptoms of depression, symptoms related to mental disorders, sleep disorders, aphasia and communication deficiencies (like problems in understanding others or being understood by others), daily pain, and feeling of loneliness [1-4, 6, 8, 9, 11, 12, 24-27]. We mainly focused on testing if BS, UR or staff's persistent frustration in contact with a resident were independent factors determining the use of psychotropic drugs from certain medicine groups. Separate logistic regression models were created for treatment interventions with antipsychotics, antidepressants and sedatives. A multivariate regression model was developed starting from a univariate model including only the variable representing the potential reason for prescribing particular type of medicine - the so-called base variable. Then, other variables were added to the model one by one, and interaction of each of them with the base variable was tested. Significant interactions were retained in the model. When all analyzed variables were added to the model, they were 
removed one by one, starting from the least significant one (with $p$ value closest to 1 ). In the final regression models, all variables were retained for which main effect was significant at a level of less than 0.1 or their presence in the model caused that main effect for some other variable was significant at that level. Each regression model was adjusted for gender, age and CPS score. The obtained results were considered statistically significant if $p$ value was less than 0.05 . All the analyses were conducted using IBM SPSS Statistics 24 software for Windows (IBM SPSS Statistics, IBM Corporation, Chicago, IL).

\section{Results}

\section{Study group characteristics}

The study group included 301 LTCF residents with a median age of 79 years (quartiles Q1 $=67, \mathrm{Q} 3=86$ ) and a female predominance $(68.4 \%)$. More than $80 \%$ of the studied residents had moderate to severe dependency in ADL and almost $70 \%$ of them showed cognitive impairment. The prevalence of any of BS was around $22.9 \%$ in the studied group, while $25.9 \%$ of LTCF residents presented at least one type of UR. The in-depth characteristics of the study group are presented in Table 1 in the column with results for total sample.

\section{The frequency of application of pharmacological and non-pharmacological interventions}

In general, the rates of the use of psychotropic drugs in our study population were high for antipsychotics (in 38.9\% of residents) and sedatives (in $35.2 \%$ of residents). About $16.9 \%$ of assessed residents received antidepressants (Table 1). The patients with BS were administered antipsychotic drugs more frequently than those without these signs. Both the presence of depression symptoms as well as other psychiatric diseases affecting residents were associated with significantly more frequent use of drugs from all considered groups (antipsychotics, sedatives, antidepressants). The residents with sleep disorders were administered sedatives and antipsychotics more frequently compared to those without such problems. The residents with daily pain received sedatives more often than those without or with less frequent than daily pain. Over one-fourth of residents expressing feeling of loneliness as well as residents with moderate cognitive impairment received antidepressants. The other considered variables and UR or frustration of the LTCF staff caused by a resident had no impact on prescribing psychotropic drugs.

Among the non-pharmacological interventions shown in Table 2, the psychological care was the most common therapy applied to $64.5 \%$ of LTCF residents. It was significantly more often prescribed to residents manifesting psychiatric disorder, depression symptoms, BS, sleep disorders and feeling loneliness, but not to residents with any type of UR. The use of PT (16.9\%), OT (3.3\%), and ADL training (15.6\%) was definitely less frequent than pharmacological treatment. These therapies were significantly more frequently applied to residents cognitively intact, with no physical ADL dependency or communication deficiencies (such as problems with understanding others or being understood by others).

\section{Factors associated with a type of pharmacological intervention}

\section{Use of antipsychotics}

As a result of the multivariate analysis (presented in Table 3), we found that the residents with BS had more than three times greater odds for receiving the antipsychotic drug than the residents without those symptoms. Similarly, a history of mental illness or a current diagnosis of a psychiatric disorder (other than depression and dementia) increased their chances of being prescribed an antipsychotic medicine. The relation between psychotic signs and the use of antipsychotics was more complex due to the interaction of two variables: psychotic symptoms and communication deficiencies. Thus, the presence of psychotic symptoms in patients who communicated well increased odds for treatment with antipsychotics about five times compared to psychotic symptoms in residents with communication problems. Neither UR nor staff's persistent frustration had influence on the prescribing of antipsychotics.

\section{Use of sedatives}

We found that sleep disorders increased more than five times the odds for the use of sedative drugs among residents compared to individuals without these problems (Table 4). The presence of BS among the younger residents without depression who did not frustrate the staff was associated with about ten times lower chance of being prescribed a sedative in comparison to individuals without BS. However, when BS appeared in older residents, the odds for taking sedative drugs increased among individuals at the age of 71-85 years and older, as compared to younger individuals. Depression among residents without BS had no influence on the use of sedatives, but when depression symptoms were accompanied with BS, the chance of using sedative drugs increased over five times compared to residents without BS. Interestingly, staff's frustration concerning the residents who showed BS was associated with ten times lower chance of prescribing sedative medicine, as compared to the impact of staff's frustration addressed at patients without BS. 
Table 1 The use of psychotropic drugs in LTCF residents in relation to their characteristics, health issues and behavioral symptoms or unsettled relationships

\begin{tabular}{|c|c|c|c|c|}
\hline \multirow[t]{2}{*}{ Characteristics of LTCF residents } & \multirow{2}{*}{$\begin{array}{l}\text { Total sample } \\
\%(n)\end{array}$} & \multicolumn{3}{|c|}{ Pharmacological intervention utilization } \\
\hline & & Antipsychotics \% (n) & Antidepressants \% (n) & Sedatives $\%(n)$ \\
\hline Use of psychotropic drugs in total study sample $(n=301)$ & $100 \%(301)$ & $38.9 \%(117)$ & $16.9 \%(51)$ & $35.2 \%(106)$ \\
\hline \multicolumn{5}{|l|}{ Basic functional characteristics } \\
\hline \multicolumn{5}{|l|}{ Age $(n=301)$} \\
\hline $31-70$ years & $31.6 \%(95)$ & $41.1 \%(39)$ & $20.0 \%(19)$ & $41.1 \%(39)$ \\
\hline $71-85$ years & $41.2 \%(124)$ & $37.1 \%(46)$ & $16.1 \%(20)$ & $32.3 \%(40)$ \\
\hline$>85$ years & $27.2 \%(82)$ & $39.0 \%(32)$ & $14.6 \%(12)$ & $32.9 \%(27)$ \\
\hline \multicolumn{5}{|l|}{ Gender $(n=301)$} \\
\hline Male & $31.6 \%(95)$ & $38.9 \%(37)$ & $14.7 \%(14)$ & $37.9 \%(36)$ \\
\hline Vs female & $68.4 \%(206)$ & $38.8 \%(80)$ & $18.0 \%(37)$ & $34.0 \%(70)$ \\
\hline \multicolumn{5}{|l|}{ ADL dependency $(n=287)$} \\
\hline No & $19.9 \%(57)$ & $28.1 \%(16)$ & $14.0 \%(8)$ & $40.4 \%(23)$ \\
\hline Moderate & $17.1 \%(49)$ & $49.0 \%(24)$ & $18.4 \%(9)$ & $38.8 \%(19)$ \\
\hline Severe & $63.1 \%(181)$ & $39.8 \%(72)$ & $16.0 \%(29)$ & $31.5 \%(57)$ \\
\hline \multicolumn{5}{|l|}{ Cognitive impairment $(n=290)$} \\
\hline No & $30.7 \%(89)$ & $29.2 \%(26)$ & $13.5 \%(12)^{*}$ & $41.6 \%(37)$ \\
\hline Moderate & $26.2 \%(76)$ & $43.4 \%(33)$ & $26.3 \%(20)$ & $39.5 \%(30)$ \\
\hline Severe & $43.1 \%(125)$ & $43.2 \%(54)$ & $13.6 \%(17)$ & $29.6 \%(37)$ \\
\hline \multicolumn{5}{|l|}{ Social functioning } \\
\hline \multicolumn{5}{|l|}{ Behavioral symptoms $(n=284)$} \\
\hline Any & $22.9 \%(65)$ & $58.5 \%(38) * * *$ & $13.8 \%(9)$ & $35.4 \%(23)$ \\
\hline Vs none & $77.1 \%(219)$ & $33.8 \%(74)$ & $17.8 \%(39)$ & $35.2 \%(77)$ \\
\hline \multicolumn{5}{|l|}{ Unsettled relationships $(n=293)$} \\
\hline Any & $25.9 \%(76)$ & $46.1 \%(35)$ & $18.4 \%(14)$ & $36.8 \%(28)$ \\
\hline Vs none & $74.1 \%(217)$ & $36.4 \%(79)$ & $16.6 \%(36)$ & $34.6 \%(75)$ \\
\hline \multicolumn{5}{|l|}{ Staff's persistent frustration in contact with resident $(n=293)$} \\
\hline Yes & $17.1 \%(50)$ & $46.0 \%(23)$ & $20.0 \%(10)$ & $44.0 \%(22)$ \\
\hline Vs none & $82.9 \%(243)$ & $37.4 \%(91)$ & $16.5 \%(40)$ & $33.3 \%(81)$ \\
\hline \multicolumn{5}{|l|}{ Chronic diseases and symptoms } \\
\hline \multicolumn{5}{|l|}{ Depression symptoms $(n=290)$} \\
\hline Yes & $33.4 \%(97)$ & $47.4 \%(46)^{*}$ & $26.8 \%(26)^{* *}$ & $43.3 \%(42)^{*}$ \\
\hline Vs no & $66.6 \%(193)$ & $34.7 \%(67)$ & $13.0 \%(25)$ & $31.1 \%(60)$ \\
\hline \multicolumn{5}{|l|}{ Psychiatric disease except depression and dementia $(n=301)$} \\
\hline Yes & $14.3 \%(43)$ & $65.1 \%(28) * * *$ & $27.9 \%(12)^{*}$ & $48.1 \%(21)^{*}$ \\
\hline Vs no & $85.7 \%(258)$ & $34.5 \%(89)$ & $15.1 \%(39)$ & $32.9 \%(85)$ \\
\hline \multicolumn{5}{|l|}{ History of mental illness $(n=298)$} \\
\hline Yes & $19.8 \%(59)$ & $66.1 \%(39) * * *$ & $23.7 \%(14)$ & $42.4 \%(25)$ \\
\hline Vs no & $80.2 \%(239)$ & $32.6 \%(78)$ & $15.5 \%(37)$ & $33.1 \%(79)$ \\
\hline \multicolumn{5}{|l|}{ Current psychotic symptoms $(n=294)$} \\
\hline Any & $35.7 \%(105)$ & $47.6 \%(50)^{*}$ & $17.1 \%(18)$ & $33.3 \%(35)$ \\
\hline Vs none & $64.3 \%(189)$ & $34.4 \%(65)$ & $16.9 \%(32)$ & $37.0 \%(70)$ \\
\hline \multicolumn{5}{|l|}{ Daily pain $(n=301)$} \\
\hline No daily pain & $92.4 \%(278)$ & $38.5 \%(107)$ & $15.8 \%(44)$ & $33.1 \%(92)^{* *}$ \\
\hline Vs daily pain & $7.6 \%(23)$ & $43.5 \%(10)$ & $30.4 \%(7)$ & $60.9 \%(14)$ \\
\hline \multicolumn{5}{|l|}{ Sleep disorders_insomnia $(n=300)$} \\
\hline Yes & $28.7 \%(86)$ & $48.8 \%(42)^{*}$ & $19.8 \%(17)$ & $59.3 \%(51) * * *$ \\
\hline Vs. no & $71.3 \%(214)$ & $35.0 \%(75)$ & $15.9 \%(34)$ & $25.7 \%(55)$ \\
\hline Aphasia $(n=297)$ & & & & \\
\hline
\end{tabular}


Table 1 (continued)

\begin{tabular}{|c|c|c|c|c|}
\hline \multirow[t]{2}{*}{ Characteristics of LTCF residents } & \multirow{2}{*}{$\begin{array}{l}\text { Total sample } \\
\%(n)\end{array}$} & \multicolumn{3}{|c|}{ Pharmacological intervention utilization } \\
\hline & & Antipsychotics \% (n) & Antidepressants \% (n) & Sedatives $\%(n)$ \\
\hline Yes & $14.5 \%(43)$ & $30.2 \%(13)$ & $18.6 \%(8)$ & $25.6 \%(11)$ \\
\hline Vs. no & $85.5 \%(254)$ & $40.2 \%(102)$ & $16.1 \%(41)$ & $37.4 \%(95)$ \\
\hline \multicolumn{5}{|c|}{ Problems with understanding others $(n=293)$} \\
\hline Yes & $44.7 \%(131)$ & $39.7 \%(52)$ & $14.5 \%(19)$ & $31.3 \%(41)$ \\
\hline Vs. no & $55.3 \%(162)$ & $38.3 \%(62)$ & $19.1 \%(31)$ & $38.3 \%(63)$ \\
\hline \multicolumn{5}{|c|}{ Problems with being understood by others $(n=291)$} \\
\hline Yes & $48.1 \%(140)$ & $41.4 \%(58)$ & $14.3 \%(20)$ & $33.6 \%(47)$ \\
\hline Vs. no & $51.9 \%(151)$ & $37.1 \%(56)$ & $19.9 \%(30)$ & $37.7 \%(57)$ \\
\hline \multicolumn{5}{|l|}{ Feeling loneliness $(n=289)$} \\
\hline Yes & $24.2 \%(70)$ & $47.1 \%(33)$ & $27.1 \%(19)^{* *}$ & $44.3 \%(31)$ \\
\hline Vs. no & $75.8 \%$ (219) & $37.0 \%(81)$ & $13.7 \%(30)^{* *}$ & $32.4 \%(71)$ \\
\hline
\end{tabular}

Bold numbers show that stars showing the level of statistical significance refer to statistically significant difference between bolded frequencies of use of certain pharmacological or non-pharmacological therapy depends on presence or not of certain character in the LTCF residents

$P$ value: ${ }^{*} p<0.05 ; * * p<0.01 ; * * * p<0.001, A D L$ activities of daily living, $L T C F$ long term care facility

\section{Use of antidepressants}

The presence of depression symptoms was associated with higher odds for treatment with antidepressants, yet in our sample this finding was not statistically significant. However, we found a significant interaction between the presence of depression symptoms and age or gender. Therefore, among residents without depression symptoms, the older ones had lower odds for receiving antidepressant as compared to the younger residents. However, residents at the age of 71 years and older showing depression symptoms were over six times more likely to be prescribed antidepressants, compared to the younger residents. In the group of residents without depression, females had a greater chance of getting the antidepressant medicine than males. Yet, in the group of residents showing signs of depression, women had significantly a lower chance of being treated with an antidepressant $(\mathrm{OR}=0.12)$ than men, compared to the group of residents without depression. The residents with mild or moderate cognitive impairment had an over two times higher chance of being treated with an antidepressant drug compared to the intact residents. Neither BS nor UR nor staff's persistent frustration had influence on the prescribing of antidepressants (see Table 5).

\section{Discussion}

As a result of our study, we found that the use of antipsychotics and sedatives in LTCF residents is substantial (38.9\% and $35.2 \%$, respectively). This observation is in line with the common trend in caring for older patients, as many systematic reviews indicated that among psychotropic drugs both the antipsychotics and sedatives are widely used in LTCFs in the USA and Europe [28-33].
Similarly to other studies $[8,10,11,32]$, our results confirmed that antipsychotics and sedatives are excessively administered to older people to manage the BS. Determining the accuracy of this treatment is difficult due to the multifactorial etiology of BS in LTCF residents (e.g., dementia, depression, psychotic symptoms, pain, type of personality, vision and hearing impairments, aphasia) [1-4, 8, 9, 12, 25, 27]. The use of antipsychotic and sedative drugs is for many LTCF individuals inevitable (especially in the short-term administration), as it decreases the BS in the course of dementia and also minimizes the risk of their own and others' lives being threatened. However, looking overall at the obtained results, it appears that BS in the course of depression is often misinterpreted for symptoms of dementia or mental disorders associated with psychotic symptoms, which leads to a more frequent use of antipsychotics and sedatives rather than antidepressants. A possible explanation for this observation lies in the complexity of mental health problems in older population that makes the diagnosis process a great challenge. There is evidence that this complexity is reflected in the pattern of pharmacological interventions where antipsychotic as well as sedative drugs are overused and antidepressants are under-prescribed $[8,10]$.

In our study, the UR (such as conflict with or repeated criticism of other residents or staff) had no impact on the use of psychotropics nor was it related to the non-pharmacological interventions. At this stage of the research, it is difficult to characterize implications of this result. On one hand, we can observe a tendency to minimize the amount of psychotropic drug used among older people due to their side effects [31-33], while on the other hand, lack of any treatment and non-pharmacological interventions may be considered as a sign of ignorance of symptoms associated with the patient's suffering. 
Table 2 The use of non-pharmacological interventions in LTCF residents in relation to their characteristics, health issues and behavioral symptoms or unsettled relationships

\begin{tabular}{|c|c|c|c|c|}
\hline \multirow[t]{2}{*}{ Characteristics of LTCF residents } & \multicolumn{4}{|c|}{ Non-pharmacological intervention utilization } \\
\hline & Physiotherapy \% (n) & $\begin{array}{l}\text { Occupational } \\
\text { therapy } \%(n)\end{array}$ & Psychological care $\%(n)$ & Training in ADL \% $(n)$ \\
\hline Study sample $(n=301)$ & $16.9 \%(51)$ & $3.3 \%(10)$ & $64.5 \%(194)$ & $15.6 \%(47)$ \\
\hline \multicolumn{5}{|l|}{ Basic functional characteristics } \\
\hline \multicolumn{5}{|l|}{ Age } \\
\hline $31-70$ years & $15.7 \%(14)$ & $3.4 \%(3)$ & $65.2 \%(58)$ & $22.5 \%(20)$ \\
\hline $71-85$ years & $17.9 \%(22)$ & $4.1 \%(5)$ & $68.9 \%(84)$ & $13.0 \%(16)$ \\
\hline$>85$ years & $18.3 \%(15)$ & $2.4 \%(2)$ & $63.4 \%(52)$ & $13.4 \%(11)$ \\
\hline \multicolumn{5}{|l|}{ Gender } \\
\hline Male & $17.8 \%(16)$ & $3.3 \%(3)$ & $62.2 \%(56)$ & $17.8 \%(16)$ \\
\hline Vs female & $17.2 \%(35)$ & $3.4 \%(7)$ & $68.0 \%(138)$ & $15.2 \%(31)$ \\
\hline \multicolumn{5}{|l|}{ ADL dependency } \\
\hline No & $35.2 \%(19)^{* * *}$ & $11.1 \%(6)^{* * *}$ & $61.1 \%(33)$ & $14.8 \%(8)^{* *}$ \\
\hline Moderate & $25.0 \%(12)$ & $6.3 \%(3)$ & $66.7 \%(32)$ & $31.3 \%(15)$ \\
\hline Severe & $11.2 \%(20)$ & $0.6 \%(1)$ & $65.0 \%(115)$ & $11.8 \%(21)$ \\
\hline \multicolumn{5}{|l|}{ Cognitive impairment } \\
\hline No & $38.1 \%(32) * * *$ & $7.1 \%(6)^{*}$ & $67.9 \%(57)$ & $17.9 \%(15)$ \\
\hline Moderate & $18.9 \%(14)$ & $4.1 \%(3)$ & $66.2 \%(49)$ & $21.6 \%(16)$ \\
\hline Severe & $4.0 \%(5)$ & $0.8 \%(1)$ & $64.5 \%(80)$ & $12.8 \%(16)$ \\
\hline \multicolumn{5}{|l|}{ Social functioning } \\
\hline \multicolumn{5}{|l|}{ Behavioral symptoms } \\
\hline Any & $16.9 \%(11)$ & $3.1 \%(2)$ & $75.4 \%(49)^{*}$ & $12.3 \%(8)$ \\
\hline Vs none & $18.4 \%(39)$ & $3.8 \%(8)$ & $61.1 \%(129)$ & $17.5 \%(37)$ \\
\hline \multicolumn{5}{|l|}{ Unsettled relationships } \\
\hline Any & $23.3 \%(17)$ & $5.5 \%(4)$ & $70.8 \%(51)$ & $24.7 \%(18)^{*}$ \\
\hline Vs none & $16.0 \%(34)$ & $2.8 \%(6)$ & $63.8 \%(136)$ & $13.6 \%(29)$ \\
\hline \multicolumn{5}{|c|}{ Staff's persistent frustration in contact with resident } \\
\hline Yes & $22.9 \%(11)$ & $8.3 \%(4)$ & $68.8 \%(33)$ & $25.0 \%(12)$ \\
\hline Vs. none & $16.8 \%(40)$ & $2.5 \%(6)$ & $65.0 \%(154)$ & $14.7 \%(35)$ \\
\hline \multicolumn{5}{|l|}{ Chronic diseases and symptoms } \\
\hline \multicolumn{5}{|l|}{ Depression symptoms } \\
\hline Yes & $15.6 \%(15)$ & $6.3 \%(6)$ & $\mathbf{7 8 . 1 \%}(\mathbf{7 5})^{* * *}$ & $24.0 \%(23)^{* * *}$ \\
\hline Vs. No & $19.3 \%(36)$ & $2.1 \%(4)$ & $59.1 \%(110)$ & $12.3 \%(23)$ \\
\hline \multicolumn{5}{|c|}{ Psychiatric disease except depression and dementia } \\
\hline Yes & $20.9 \%(9)$ & $7.0 \%(3)$ & $85.7 \%(36)^{* *}$ & $34.9 \%(15) * * *$ \\
\hline Vs. no & $16.7 \%(42)$ & $2.8 \%(7)$ & $62.9 \%(158)$ & $12.7 \%(32)$ \\
\hline \multicolumn{5}{|l|}{ History of mental illness } \\
\hline Yes & $10.2 \%(6)$ & $6.8 \%(4)$ & $76.3 \%(45)$ & $23.7 \%(14)$ \\
\hline Vs. no & $19.4 \%(45)$ & $2.6 \%(6)$ & $63.2 \%(146)$ & $14.2 \%(33)$ \\
\hline \multicolumn{5}{|l|}{ Psychotic symptoms } \\
\hline Any & $12.5 \%(13)$ & $2.9 \%(3)$ & $67.0 \%(69)$ & $22.1 \%(23)^{*}$ \\
\hline Vs. none & $20.8 \%(38)$ & $3.8 \%(7)$ & $65.6 \%(120)$ & $13.1 \%(24)$ \\
\hline \multicolumn{5}{|l|}{ Parkinson disease } \\
\hline Yes & $30.0 \%(6)$ & $5.0 \%(1)$ & $70.0 \%(14)$ & $30.0 \%(6)$ \\
\hline Vs. no & $16.5 \%(45)$ & $3.3 \%(9)$ & $65.8 \%(179)$ & $14.7 \%(40)$ \\
\hline \multicolumn{5}{|l|}{ Daily pain } \\
\hline No & $16.6 \%(45)$ & $3.3 \%(9)$ & $65.2 \%(176)$ & $16.2 \%(44)$ \\
\hline Vs any daily pain & $26.1 \%(6)$ & $4.3 \%(1)$ & $78.3 \%(18)$ & $13.0 \%(3)$ \\
\hline Sleep disorders - insomnia & & & & \\
\hline
\end{tabular}


Table 2 (continued)

\begin{tabular}{|c|c|c|c|c|}
\hline \multirow[t]{2}{*}{ Characteristics of LTCF residents } & \multicolumn{4}{|c|}{ Non-pharmacological intervention utilization } \\
\hline & Physiotherapy \% (n) & $\begin{array}{l}\text { Occupational } \\
\text { therapy } \%(n)\end{array}$ & Psychological care $\%(n)$ & Training in ADL $\%(n)$ \\
\hline Yes & $19.3 \%(16)$ & $3.6 \%(3)$ & $75.9 \%(63)^{*}$ & $12.0 \%(10)$ \\
\hline Vs. no & $16.7 \%(35)$ & $3.3 \%(7)$ & $62.2 \%(130)$ & $17.6 \%(37)$ \\
\hline \multicolumn{5}{|l|}{ Aphasia } \\
\hline Yes & $4.7 \%(2)^{*}$ & 0 & $69.8 \%(30)$ & $20.9 \%(9)$ \\
\hline Vs. no & $19.8 \%(49)$ & $4.0 \%(10)$ & $65.4 \%(161)$ & $15.4 \%(38)$ \\
\hline \multicolumn{5}{|l|}{ Problems with understanding others } \\
\hline Yes & $3.1 \%(4) * * *$ & $0.8 \%(1)^{*}$ & $66.2 \%(86)$ & $13.7 \%(18)$ \\
\hline Vs. no & $30.3 \%(47)$ & $5.8 \%(9)$ & $65.2 \%(101)$ & $18.7 \%(29)$ \\
\hline \multicolumn{5}{|c|}{ Problems with being understood by others } \\
\hline Yes & $5.8 \%(8)^{* * * *}$ & $0.7 \%(1)^{*}$ & $66.7 \%(92)$ & $15.1 \%(21)$ \\
\hline Vs. no & $29.7 \%(43)$ & $6.2 \%(9)$ & $64.8 \%(94)$ & $17.9 \%(26)$ \\
\hline \multicolumn{5}{|l|}{ Feeling loneliness } \\
\hline Yes & $15.7 \%(11)$ & $4.3 \%(3)$ & $77.1 \%(54) *$ & $24.3 \%(17)^{*}$ \\
\hline Vs. no & $18.9 \%(40)$ & $3.3 \%(7)$ & $62.1 \%$ & $14.2 \%(30)$ \\
\hline
\end{tabular}

Bold numbers show that stars showing the level of statistical significance refer to statistically significant difference between bolded frequencies of use of certain pharmacological or non-pharmacological therapy depends on presence or not of certain character in the LTCF residents $P$ value: $* p<0.05 ; * *<0.01$; *** $p<0.001, A D L$ activities of daily living, $L T C F$ long term care facility

Table 3 Factors determining the risk of prescribing antipsychotics in the LTCF residents - the results of logistic regression analysis

\begin{tabular}{lll}
\hline Characteristics of residents & \multicolumn{2}{l}{ The use of antipsychotics } \\
\cline { 2 - 3 } & OR (95\% CI) & $p$ \\
\hline Presence of any of behavioral symptoms & $3.06(1.59-5.86)$ & 0.001 \\
History of mental illness & $3.29(1.59-6.81)$ & 0.001 \\
Current psychiatric disease other than depression and dementia & $2.55(1.12-5.84)$ & 0.026 \\
No communication deficiency & $0.64(0.33-1.23)$ & 0.183 \\
Presence of any psychotic symptoms** (vs none psychotic symptoms) & $0.55(0.26-1.19)$ & 0.129 \\
Presence of any psychotic symptoms** by no communication deficiencies & $5.12(1.50-17.48)$ & 0.009 \\
Constant & 0.45 & 0.003 \\
\hline
\end{tabular}

$L T C F$ long term care facility, $C I$ confidence interval, $O R$ odds ratio, $p p$ value, ref reference category *Communication deficiencies in terms of problems in understanding others or being understood by others

**Psychotic symptoms include abnormal thought process, delusions, hallucinations
Moreover, we found that LTCF staff's frustration in caring for residents with BS was associated with reduced risk of prescribing sedative drugs (Table 4). It was even stronger among residents with cognitive impairment. This observation may indicate that staff's frustration while delivering the care to residents does not trigger application of psychotropic drugs. That should be evaluated as an indicator of good quality care. Looking overall at the obtained results, it appears that the staff may be more tolerant to the presence of BS in patients with dementia than in residents without clear cause of BS, which may lead to lower psychotropic drug use in residents with cognitive impairment.

Apart from the results considering the analyzed problems with social functioning, we found interesting results showing that residents with communication deficiencies (e.g., due to stroke or dementia) manifesting psychotic signs had a lower chance of being treated with antipsychotic drugs compared to individuals with psychotic symptoms but without problems with understanding others or being understood by others. This result might be interpreted as not sufficient treatment of delusions, hallucinations and abnormal thought process in residents who are not able to communicate well.

Interestingly, neither BS nor UR nor staff's persistent frustration had influence on prescribing antidepressants. It is surprising as the results of our previous research conducted in the same study group had shown that depression was a significant risk factor for both behavioral symptoms and unsettled relationships $[25,27]$. This observation is in 
Table 4 Factors determining the risk of prescribing sedatives in the LTCF residents- the results of logistic regression analysis

\begin{tabular}{llr}
\hline Characteristics of residents & The use of sedatives & \\
\cline { 2 - 2 } & OR $(95 \%$ CI $)$ & $p$ \\
\hline Age 31-70 years (ref) & 1 & 0.071 \\
Age 71-85 years & $0.51(0.24-1.06)$ & 0.091 \\
Age $>$ 85 years & $0.50(0.23-1.12)$ & 0.001 \\
Sleep disorders vs none & $5.22(2.74-9.98)$ & 0.016 \\
Presence of any of behavioral symptoms vs none & $0.10(0.02-0.65)$ & 0.009 \\
Age 31-70 years by behavioral symptoms (ref) & 1 & 0.042 \\
Age 71-85 years by behavioral symptoms & $12.10(1.88-77.77)$ & 0.728 \\
Age $>$ 85 years by behavioral symptoms & $9.15(1.09-77.01)$ & 0.041 \\
Depression symptoms & $0.87(0.40-1.88)$ & 0.104 \\
Behavioral symptoms by depression symptoms & $5.60(1.07-29.20)$ & 0.018 \\
Staff expressed persistent frustration in caring for resident & $2.72(0.81-9.07)$ & 0.076 \\
Staff expressed persistent frustration in caring for resident by & $0.10(0.02-0.68)$ & 0.009 \\
$\quad$ behavioral symptoms & & \\
Daily pain vs none or less frequent than daily & $2.80(0.88-8.75)$ & \\
Constant & 0.48 & \\
\hline
\end{tabular}

$L T C F$ long term care facility, $C I$ confidence interval, $O R$ odds ratio, $p p$ value, ref reference category

\begin{tabular}{lll}
\hline Characteristics of residents & \multicolumn{2}{l}{ The use of antidepressants } \\
\cline { 2 - 3 } & OR $(95 \% \mathrm{CI})$ & $p$ \\
\hline Depression symptoms & $2.81(0.65-12.10)$ & 0.167 \\
Age 31-70 years (ref) & 1 & \\
Age 71-85 years & $0.21(0.07-0.64)$ & 0.006 \\
Age $>$ 85 years & $0.25(0.08-0.85)$ & 0.026 \\
Age 31-70 years by depression symptoms (ref) & 1 & 0.015 \\
Age 71-85 years by depression symptoms & $7.34(1.48-36.31)$ & 0.050 \\
Age $\geq 86$ years by depression symptoms & $6.40(0.99-41.09)$ & 0.026 \\
Female gender vs male & $3.70(1.17-11.71)$ & 0.008 \\
Gender by depression symptoms & $0.12(0.02-0.56)$ & \\
No cognitive impairment or nearly intact (ref) & 1 & 0.051 \\
Mild or moderate cognitive impairment & $2.33(0.99-5.45)$ & 0.964 \\
Moderate severe to very severe cognitive impairment & $0.98(0.42-2.28)$ & 0.083 \\
Daily pain vs none or less frequent than daily & $2.56(0.88-7.39)$ & 0.000 \\
Constant & 0.102 & \\
\hline
\end{tabular}

$L T C F$ long term care facility, $C I$ confidence interval, $O R$ odds ratio, $p p$ value, $r e f$ reference category
Table 5 Factors determining the risk of prescribing antidepressants in the LTCF residents-the results of logistic regression analysis sync with the results of other studies [1-4, 11, 24]. The lack of administration of antidepressants therapy among residents with BS and UR could be explained with the common underestimation of depression symptoms presented by older patients. Existing evidence suggests that as much as $85 \%$ of depressed older people are not diagnosed properly [6], and therefore treated in an inadequate way.

In comparison with pharmacotherapy, the non-pharmacological interventions were rarely applied, mostly to the relatively independent in ADL and not cognitively impaired LTCF residents. The most common type was the psychological care, prescribed to residents with different psychiatric disorders, BS, sleep disturbance and feeling of loneliness. In the analyzed LTCF, the psychological care was tailored to the residents' needs and included life review, validation therapy, reality orientation, reminiscence, supportive counseling. In the literature review, such psychological interventions are listed as effective in the reduction of BS, through the use of an appropriate communication method and emotional support [8].

The results obtained in our study confirmed the complexity of diagnosing and treating the LTCF residents with BS and UR, due to interaction and overlap of various symptoms and factors related to the functional status. 
To maximize the effectiveness in managing residents' problems with social functioning, it is crucial to differentiate their possible determinants including multifactorial etiology of BS and UR in older residents $[1-4,8,9$, $12,25,27]$. The differential diagnosis should take into account relations between dementia and depression, pain and depression, psychosis and depression or dementia, the coexistence of sleep disturbances, pain and social isolation. Furthermore, the complexity of mental health problems in LTCF population points to the need of systematic examination of residents in LTC institutions for depression as well as psychotic and behavioral symptoms. Such observations should be recorded in the daily routine care reports on residents functioning. Based on our findings, we recommend that LTC staff should be trained in effective recognition and understanding of possible causes of BS and UR in LTCF residents, including depression, pain, sensory deficits, cognitive impairment, sleep disorders, feeling of loneliness, psychosis and limitations of autonomy with physical or chemical restraints $[1-4,8,9$, $12,25,27]$. It is also very important to take care of minimizing the caregiver's job stress, as caring for residents with BS and UR in long-term perspective is demanding, resulting in higher risk of absenteeism and burnout syndrome in LTCF staff $[10,26]$. Since medications and nonpharmacological interventions rarely totally eliminate the presence of BS and UR in LTCF residents [5, 7, 11], it is crucial to provide the staff with debriefing meetings which have good effects on managing work stress and delivering supportive counseling.

\section{Study advantages and limitations}

This article aimed to scrutinize the line of accepted treatment of typical clinically complex LTCF population (not limited to residents with dementia) presenting BS and UR. The value of this study is that the data collection was solely intended for research purposes, was performed by trained staff and covered all residents in the facility. The data on treatment was accurate and obtained based on drug dispensary cards which included name and daily dose of drugs administered on the day of data collection. However, due to the cross-sectional design of the study, its findings do not indicate cause-and-effect relationships. Thus, we could not analyze the effects of pharmacological and non-pharmacological approaches. We focused on studying the relations between symptoms and the type of treatment looking for the most probable choice of therapeutic pattern. The future research on pharmacological and non-pharmacological interventions in managing BS and UR in LTCF residents should undertake an observational approach to study the issue of individualization and proper selection of treatment as well as to register potential adverse effects. Moreover, the characteristics of pharmacological and non-pharmacological interventions should be analyzed to optimize their impact, including timing, duration, location and intensity.

\section{Conclusions}

To sum up, the main goal of this paper was to find if BS and UR presented by the LTCF residents increase the risk of treatment with certain classes of psychotropic drugs. We found that residents with BS (such as wandering, socially inappropriate behaviors, verbal and physical abuse, resistance to prescribed care or treatment) were preferably treated with antipsychotics and sedatives rather than antidepressants, even when BS were associated with depression. Furthermore, we did not find any significant relation between UR and taking psychotropic drugs. It was also concluded that LTCF staff's frustration in caring for residents with BS did not trigger more frequent administering of psychotropic medicine.

The obtained results constitute confirmation that the care and treatment of LTCF residents with BS and UR is a complex and challenging task. Since both BS and UR may appear in the course of different diseases, we concluded that multifactorial etiology of these symptoms in clinically complex LTCF residents requires better recognition of the underlying causes for applying an appropriate treatment. We believe the results of our study may encourage care professionals to pay more attention to finding causes of BS presented by the LTCF residents and contribute to improving their strategy in managing these symptoms.

Acknowledgements The study was funded by the Jagiellonian University Medical College Grant no K/ZDS/003797.

\section{Compliance with ethical standards}

Conflict of interest KS and UH declare no conflict of interest.

Ethical approval The authors declare that the study has been registered and approved by the Jagiellonian University Ethics Committee and it was conducted in line with the current laws, meeting the standard ethical requirements.

Informed consent Informed consent has been obtained from the residents or their next of kin to be kept in the facility, thus the researchers have performed the analysis on the anonymous data having no access to any personal data.

Open Access This article is distributed under the terms of the Creative Commons Attribution 4.0 International License (http://creativeco mmons.org/licenses/by/4.0/), which permits unrestricted use, distribution, and reproduction in any medium, provided you give appropriate 
credit to the original author(s) and the source, provide a link to the Creative Commons license, and indicate if changes were made.

\section{References}

1. Isaksson U, Äström S, Sandman PO, Karlsson S (2008) Factors associated with the prevalence of violent behavior among residents living in nursing homes. J Clin Nurs 18:972-980

2. Gruber-Baldini AL, Boustani M, Sloane PD, Zimmerman S (2004) Behavioral symptoms in residential care/assisted living facilities: prevalence, risk factors, and medication management. J Am Geriatr Soc 52:1610-1617

3. Koopmans RT, Zuidema SU, Leontjevas R, Gerritsen DL (2010) Comprehensive assessment of depression and behavioral problems in long-term care. Int Psychogeriatr 22:1054-1062

4. Tsai CF, Ouyang WC, Chen LK, Lan CF, Hwang SJ, Yang CH, Su TP (2009) Depression is the strongest independent risk factor for poor social engagement among Chinese elderly veteran assistedliving residents. J Chin Med Assoc 72:478-483

5. Cohen-Mansfield J, Werner P, Culpepper WJIII, Wolfson MA et al (1996) Wandering and aggression. In: Carstensen LL, Edelstein BA, Dornbrand L (eds) The practical handbook of clinical gerontology. Sage Publications, California, pp 374-397

6. Onder G, Carpenter I, Finne-Soveri H, Gindin J, Frijters D, Henrard JC et al (2012) Assessment of nursing home residents in Europe: the services and health for elderly in long term care (SHELTER) study. BMC Health Serv Res. 12:5. https://doi. org/10.1186/1472-6963-12-5

7. Soreff S (2012) In: Olisah V (ed) Essential notes in psychiatry. IntechOpen, London., pp 221-242. https://doi.org/10.5772/36738

8. Voyer P, Verreault R, Azizah G, Desrosiers J, Champoux N (2005) Bedard A (2005) Prevalence of physical and verbal aggressive behaviors and associated factors among older adults in long-term care facilities. BMC Geriatr 5:13. https://doi. org/10.1186/1471-2318-5-13

9. Talerico KA, Evans LK, Strumpf NE (2002) Mental health correlates of aggression in nursing home residents with dementia. Gerontol 42:169-177

10. Voyer P, Martin LS (2003) Improving geriatric mental health nursing care: making a case for going beyond psychotropic medications. Int J Ment Health Nurs 12:11-21

11. Snowden M, Sato K, Roy-Byrne P (2003) Assessment and treatment of nursing home residents with depression or behavioral symptoms associated with dementia: a review of the literature. $\mathrm{J}$ Am Geriatr Soc 51:1305-1317

12. Liebson E (2001) Aggression in dementia. Home Health Care Consult 8:26-31

13. Cohen-Mansfield J (2001) Non-pharmacologic interventions for inappropriate behaviors in dementia: a review, summary and critique. Am J Geriatr Psychiatry 9:361-381

14. Verrusio W, Renzi A, Cecchetti F, Gaj F, Coi M, Ripani M, Cacciafesta M (2018) The effect of a physical training with the use of an exoskeleton on depression levels in institutionalized elderly patients: a pilot study. J Nutr Health Aging. https://doi. org/10.1007/s12603-018-1044-2

15. Heyn P, Abreu BC, Ottenbacher KJ (2004) The effects of exercise training on elderly persons with cognitive impairment and dementia: a meta-analysis. Arch Phys Med Rehabil 85:1694-1704
16. Singh NA, Clements KM, Singh MA (2001) The efficacy of exercise as a long-term antidepressants in elderly subjects: a randomized, controlled trial. J Gerontol A Biol Sci Med Sci 56:M497-M504

17. Gerdner LA (2000) Effects of individualized vs classical relaxation music on the frequency of agitation in elderly persons with Alzheimer's disease and related disorders. Int Psychogeriatr 12:49-65

18. Hirdes JP, Ljunggren G, Morris JN, Frijters DHM, Finne-Soveri $\mathrm{H}$, Gray L et al (2008) Reliability of the InterRAI suite of assessment instruments: a 12-country study of an integrated health information system. BMC Health Serv Res 8:277. https://doi. org/10.1186/1472-6963-8-277

19. Morris JN, Fries BE, Mehr DR, Hawes C, Phillips C, Mor V, Lipsitz LA (1994) The MDS cognitive performance scale. J Gerontol 49:M174-M182

20. Burrows AB, Morris JN, Simon SE, Hirdes JP, Phillips C (2000) Development of a minimum data set-based depression rating scale for use in nursing homes. Age Ageing 29:165-172

21. Morris JN, Fries BE, Morris SA (1999) Scaling ADLs within the MDS. J Gerontol A Biol Sci Med Sci 54:M546-M553

22. Fries BE, Simon SE, Morris JN, Flodstrom C, Bookstein FL (2001) Pain in U.S. nursing homes: validating a pain scale for the minimum data set. Gerontologist 41:173-179

23. WHO Collaborating Centre for Drug Statistics Methodology, Guidelines for ATC classification and DDD assignment 2013. Oslo 2012

24. Achterberg W, Pot AM, Kerkstra A, Ooms M, Muller M, Ribbe $M$ (2003) The effect of depression on social engagement in newly admitted Dutch nursing home residents. Gerontologist 43:213-218

25. Horwath U, Szczerbińska K (2017) Factors associated with unsettled relationships between residents and care staff in long-term care facility. Eur Geriatr Med 8:460-466

26. Isaksson U, Graneheim UH, Richter J, Eisemann M, Äström S (2008) Exposure to violence in relation to personality traits, coping capabilities, and burnout among caregivers in nursing homes: a case-control study. Scand J Caring Sci 22:551-559

27. Pawłucka U, Brzyski P, Kubicz D, Szczerbińska K (2016) The determinants of behavioral symptoms in long-term care facility residents. Eur Geriatr Med 7:157-162

28. Alanen HM, Finne-Soveri H, Noro A, Leinonen E (2006) Use of antipsychotics among nonagenarian residents in long-term institutional care in Finland. Age Ageing 35:508-513

29. Aparasu RR, Mort J, Sitzman S (1998) Psychotropic prescribing for the elderly in office-based practice. Clin Therap 20:603-616

30. Lanctot KL, Best TS, Mittmann N, Liu BA, Oh PI, Einarson TR, Naranjo CA (1998) Efficacy and safety of neuroleptics in behavioral disorders associated with dementia. J Clin Psychiatry 59:550-561

31. Lucchetti G, Lucchetti AL (2017) Inappropriate prescribing in older persons: a systematic review of medications available in different criteria. Arch Gerontol Geriatr 68:55-61

32. Morin L, Laroche ML, Texier G, Johnell K (2016) Prevalence of potentially inappropriate medication use in older adults living in nursing homes: a systematic review. J Am Med Dir Assoc 17:862. e1-862.e9

33. Storms H, Marquet K, Aertgeerts B, Claes N (2017) Prevalence of inappropriate medication use in residential long-term care facilities for the elderly. Eur J Gen Pract 23:69-77 